\title{
Osteometabolic profile: a possible tool in decision making for implant rehabilitation in patients under antiresorptive drugs
}

\author{
Gianluca Colapinto ${ }^{1}$, Funda Goker ${ }^{2}$, Massimo Del Fabbro², Tiziano Testori $^{2}$ \\ 1 ORAL MED CARE srl \\ 2 University of Milan \\ Funding: The author(s) received no specific funding for this work. \\ Potential competing interests: The author(s) declared that no potential competing interests exist.
}

\section{Abstract}

This comparative study included 146 patients that are under risk of developing MRONJ and needed oral rehabilitation with implant-supported prosthesis. All the included patients had metabolic profile assessments and were divided into two groups: G1-normal osteometabolic profile (92 patients), G2- altered osteometabolic profile (54 patients). All patients in G1, regardless of gender and type of prosthetic rehabilitation, showed a regular osseointegration process without any complications. However, 42 patients in G2 (77\%) were diagnosed with MRONJ within six months afterimplant insertions, which did not compromise implant osseointegration and prosthetic rehabilitation. In other 12 patients (23\%) in adjunct to ONJ development, implants did not osseointegrate. All patients with ONJ were successfully treated according to the SISBO pharmacological protocol.According to the results of this study, osteometabolic profile assessment can be considered a useful tool in terms of decreasing the risk of developing ONJ in patients.

BACKGROUND: All drugs associated with the development of osteonecrosis of the jaws (ONJ), independent of their action mechanism, interfere with normal bone metabolism and healing. Implant insertion, and the consequent osseointegration process, may represent a risk factor for the onset of $\mathrm{ONJ}$ in patients under antiresorptive drugs, who may undergo early or late implant loss and compromission of prosthetic rehabilitation $(1,2)$. Since bone metabolism can be seriously altered in these subjects, aim of the present study was to evaluate whether the patient's osteometabolic profile (serum levels of calcium, PTH, Vitamin D, Osteocalcin, CTX) is related to the success of implant osseointegration. METHODS: In this comparative study patients under antiresorptive drugs and in need for implant treatment, referred to the Oral Med Care Center, Bari, Italy, by the IRCCS Giovanni Paolo II Oncological Institute, by freelance colleagues, and by the University Hospital of the Polyclinic of Bari, were selected. After investigation of the medical history, a clinical and radiological evaluation, patients were informed about the possible risk of developing ONJ in relation to implant surgery. After obtaining informed consent, the patients who met the following inclusion criteria were selected for this study: 1) 
treatment with oral bisphosphonates (alendronate $70 \mathrm{mg} / \mathrm{week}$ ) for at least 5 consecutive years, or a denosumab administration (60 mcg/6 months) because of osteoporosis, 2) absence of other pathologies that could affect metabolic bone values, 3) need for oral rehabilitation through implant-supported mandibular and/or maxillary overdenture, 4) implant insertion with submerged technique, 5) blood tests for the bone metabolic profile, performed at the time of implant surgery, at the Hematology Laboratory of Hospital "F. Miulli" Acquaviva delle Fonti, Italy, before the implant rehabilitation. 6) 5 year follow up. Included patients were divided in two groups: one with a normal osteometabolic profile, and another with altered profile (consisting of the following serum levels: PTH $>104 \mathrm{pg} / \mathrm{ml}$, Vitamin $\mathrm{D}<18 \mathrm{ng} / \mathrm{ml}$, Osteocalcin $<10 \mathrm{ng} / \mathrm{ml}$, CTX $<90$ $\mathrm{pg} / \mathrm{ml})$. Recruitment was stopped when at least 50 patients for group were treated.

RESULTS: 146 patients were included: 113 women, of which $73 \%$ treated with alendronate, and $27 \%$ with denosumab (81 with maxillary overdenture, 22 mandibular overdenture and 10 with both rehabilitations); 33 men, of which $87 \%$ treated with alendronate and $13 \%$ with denosumab (16 with maxillary overdenture, 12 with mandibular overdenture and 5 with both types). Ninety-two patients (63\% of the total sample) had a normal osteometabolic profile. Among patients treated with denosumab, it had been taken more than six months earlier. All patients of this group, regardless of gender and type of prosthetic rehabilitation, showed a regular osseointegration process without healing complications. Fifty-four patients (37\%) displayed an altered metabolic profile. In patients who had taken denosumab earlier compared to the blood test date the alteration was more pronounced. Regardless of the drug taken, the gender and the type of prosthetic rehabilitation, 42 patients of this group (77\%) were diagnosed with osteonecrosis of the jaws (classified according to the AAOMS criteria) within six months from implant insertion, which did not compromise implant osseointegration and prosthetic rehabilitation. In other 12 patients (23\%) in adjunct to ONJ development, implants did not osseointegrate. All patients with ONJ were successfully treated according to the SISBO pharmacological protocol (3), in order to restore markers' levels within normal values.

CONCLUSIONS: Based on the results of this study, the evaluation of the osteometabolic profile can be considered a useful tool in evaluating the risk of developing $\mathrm{ONJ}$ in patients candidate to implant therapy, who have a history of antiresorptive therapy for osteoporosis. ${ }^{[1][2][3]}$

\section{References}

1. 'Viviann Ruocco-Vetucci, Ana Paula de Souza Faloni, Rafael Silveira Faeda. (2019). Follow-Up of an ImplantSupported Rehabilitation After Long-Term Use of Alendronate. Journal of Craniofacial Surgery, vol. 30 (8), e793-e796. doi:10.1097/scs.0000000000005782.

2. ^Andreas Stavropoulos, Kristina Bertl, Peter Pietschmann, Nikolaos Pandis, et al. (2018). The effect of antiresorptive drugs on implant therapy: Systematic review and meta-analysis. Clin Oral Impl Res, vol. 29, 54-92. doi:10.1111/clr.13282.

3. 'Gianluca Colapinto, Raffaele Volpi, Giovanni Forino, Vito Tricarico, et al. (2018). Patients' osteometabolic control improves the management of medication-related osteonecrosis of the jaw. Oral Surgery, Oral Medicine, Oral Pathology and Oral Radiology, vol. 125 (2), 147-156. doi:10.1016/j.00oo.2017.10.015. 
